\section{MarsCAPE: Mars Communicated Through an Augmented, Physical Environment}

James Sprinks $^{1}$, Liz Dowthwaite ${ }^{2}$, Gary Priestnall ${ }^{3}$, Jessica Wardlaw $^{4}$

\author{
${ }^{1}$ School of Engineering, Nottingham Trent University, \\ Nottingham, UK, james.sprinks@ntu.ac.uk \\ ${ }^{2}$ Horizon Digital Economy Research Institute, University of \\ Nottingham, Nottingham, UK, \\ liz.dowthwaite@nottingham.ac.uk \\ ${ }^{3}$ School of Geography, University of Nottingham, Nottingham, \\ UK, gary.priestnall@nottingham.ac.uk \\ ${ }^{4}$ The Natural History Museum, London, UK, \\ j.wardlaw@nhm.ac.uk
}

\section{Keywords}

D.2.14.a User Interfaces, H.1.2.a Human Factors, I.4.0.a Image Displays, I.6.9.g Visualization Techniques and Methodologies

\begin{abstract}
In the last decade, vast amounts of planetary science data has been made available publicly often focused on Mars. Such data is typically disseminated via the web and made available through screen-based visualisations. However, this approach can make it difficult to convey the broader context of a feature of interest or the spatial arrangement of surface phenomena. To better support learning and engagement, we present and evaluate MarsCAPE: Mars Communicated through an Augmented, Physical Environment. MarsCAPE consists of physical models of the surface of Mars, augmented by projected information and visualizations. To assess its learning and engagement value, a structured workshop and formal evaluation were conducted. Participants reported a significant increase in knowledge, found the models engaging, and exhibited natural learning without prompting. Systems such as MarsCAPE have potential to provide an interesting, educational way for the public to access planetary data that goes beyond the capabilities of on-screen visualizations.
\end{abstract}

\section{Introduction}

In the last decade, a spate of missions such as the New Horizons mission to Pluto [1] have arguably increased public interest in space exploration and planetary science to a level not seen since the 'Space Race' era. Mars exploration in particular has always been popular with the public, appearing prominently in literature, arts and popular culture [2]. Future missions investigating the possibility of life on Mars, such as the ExoMars mission [3], are likely to increase public interest even further.
Planetary scientists have started to realise the potential of such public interest through a range of initiatives. For example, citizen science allows public participation in scientific research, sharing the workload of analysing the vast amounts of remotely collected data. Martian science teams have developed online platforms like Planet Four [4] that allow amateur communities to contribute scientific analysis; learning is one of the key motivations of the citizen science community [5]. Through this process, citizen science platforms have become powerful tools for science communication, engagement and education [6].

Engaging public audiences in planetary science involves the visualisation of landscapes and phenomena that they have no direct experience of, providing a challenge in communicating not only their appearance but also their size and spatial arrangement. When visualising landscapes on Earth there are usually recognisable and familiar objects or patterns, which help people appreciate the scale of a landscape. When visualising a remote landscape like that of Mars, however, there is not the same 'frame of reference' for the audience, so it may be harder for them to understand what they are looking at.

Repositories of planetary science data give researchers access to imagery and digital terrain datasets that can be manipulated within remote sensing or Geographical Information System (GIS) software. Maps can be produced which synthesise and generalise some of the data coming from planetary surveys and go some way towards helping audiences interpret a landscape [7]. Interpreting physical landscapes from maps can involve a cognitive 'model building' process that can be replaced to some extent by using three-dimensional visualisation. Virtual globes such as Google Mars offer platforms for browsing imagery that are accessible to a wider audience and allow panoramic imagery taken from landing craft to be placed into the landscape context provided by the digital terrain surface. Such platforms offer excellent ways to browse data using an interface that is familiar and intuitive to many. They can also provide useful tools for teachers to develop inquiry-based exploratory learning exercises that have the potential to promote spatial thinking in students [8].

For public audiences a virtual globe interface may not provide the control required to promote a specific type of learning, or to communicate a particular message. When the context is a public space such as a visitor centre or science exhibition, there is also no need to restrict the mode of display to a screen. An option in such circumstances is to represent the surface terrain as a physical relief model and to augment that through projection, with ancillary information via audio or a monitor. A display configuration like this is referred to as a Projection Augmented Relief Model (PARM) [9].

This paper reports experiences of using PARM to engage public audiences with the nature and scale of parts of the Martian landscape. This formed part of a project called MarsCAPE (Mars Communicated through an Augmented, Physical Environment) [10]. The broad aims of the MarsCAPE project are:

1. To engage the public with space, and human endeavour in space, by highlighting the 
similarities and differences between Earth and Mars and why they exist.

2. To bring the Martian surface to life, through physical landscape models augmented with animated projection, in order to improve understanding of the appearance of the Martian surface.

3. To educate about missions to Mars and their practicalities.

4. To provide public access to planetary data through a novel, innovative display that informs and demonstrates new visualisation techniques that can be repeated for larger audiences.

To evaluate the project against these aims, MarsCAPE was presented at numerous public events for a range of audiences over an 18-month period. With each event, feedback was collected and the system refined, culminating in a summer school workshop held at the UK's National Space Centre where, importantly, a more formal evaluation of MarsCAPE's potential for learning and engagement took place. We present here the motivation for using the PARM technique and how MarsCAPE was developed, before detailing the design findings of the workshop.

\section{Approaches to Visualising Landscape}

Landscape visualisation is often used to communicate changes to a landscape or environmental conditions in the past or the future, for example showing a local community what a new development will look like, with the size and relative position shown against a familiar frame of reference. There are many approaches to visualisation in the field of landscape architecture, often involving computer generated 3D visualisation, which in turn presents various levels of dynamism and interactivity [11].

Visual representations used in landscape visualisation can present a focus for discussion through a common language [12] to which a wide range of people can relate. Good visual simulations should be understandable, convincing and unbiased [13]; creators of landscape visualisations should work to avoid producing believable and convincing visual representations that are either misleading or underpinned by erroneous data [14].

There are many options for visualising landscapes (see

Figure 1), with each technique having its own merits, costs and complexities of implementation. The choice of technique depends upon the aims of the exercise, the target audience and the time and resources available. Static images rendered from a 3D model span both physical and digital media in that they can form printed images but can also be distributed via screens and as such remain a commonly used mode of delivery for landscape visualisations. Animated fly through sequences are another popular choice as they are engaging and rich in information but still allow the creator to control the message given to viewers. Allowing interaction through virtual globes like Google Mars or systems based upon games engines is appealing to users but introduces challenges for interaction design for developers of the visualisations. The introduction of large stereo screens or head-mounted displays allows more visual immersion though often at the expense of broader spatial context.

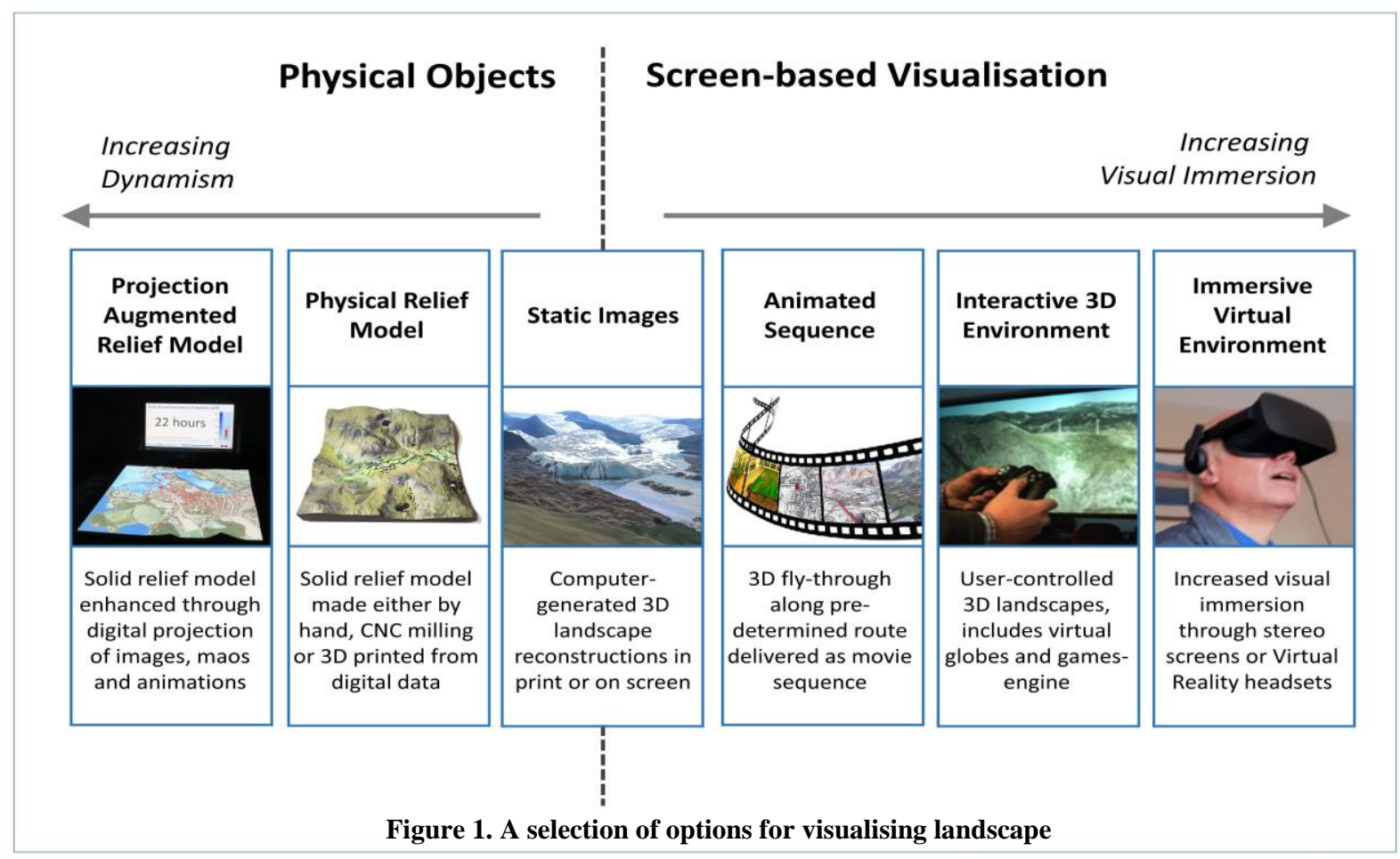


Screen-based landscape visualisation supports wider dissemination via the web or can exploit specialist immersive technology to produce realistic first-person perspectives of virtual scenes. What can be more challenging is to convey the broader landscape context of a feature of interest or the spatial arrangement and scale of landforms or phenomena. This becomes even more challenging when the landscape is unfamiliar as the viewer has little or no direct frame of reference. One method that has been used historically to convey such landscapes has been to use physical relief models. One of the earliest uses was to support military strategy by

representing remote fortifications where both buildings and the surrounding landscape were modelled in great detail, for example the French plan-reliefs [15]. The capability of relief models to convey the spatial arrangement of features is seen in their use for military training [16], in preparation for missions based in remote areas.

The use of models to communicate spatial form has been seen across the sciences [17] and benefits from the power of human stereovision to discriminate detail in three dimensions and the intuitive forms of interaction that are possible, such as holding and rotating an object to gain alternative perspectives. With a horizontally mounted relief model the viewer is free to walk around it gaining not only alternative views onto the scene but a sense of three-dimensional structure from motion around the model.

Conventional relief models are coloured to nature using textured materials and paints but with easier 3D fabrication technologies and digital projection technology it is possible to create surface models augmented with alternative texture maps. Projection Augmented Relief Models (PARM) displays feature detailed physical relief models, created using CNC milling or $3 \mathrm{D}$ printing, augmented from above with high resolution maps, imagery and animations, often with screens displaying ancillary information [9]. Situating them within the landscapes being modelled, as media rich You-Are-Here maps, has been shown to be effective. Their potential to communicate landscapes that are unfamiliar to the viewer and 'not of this Earth' has yet to be explored. Figure 2 shows a typical PARM configuration where a monitor is used to display information related to the imagery being projected onto the model at any given time.

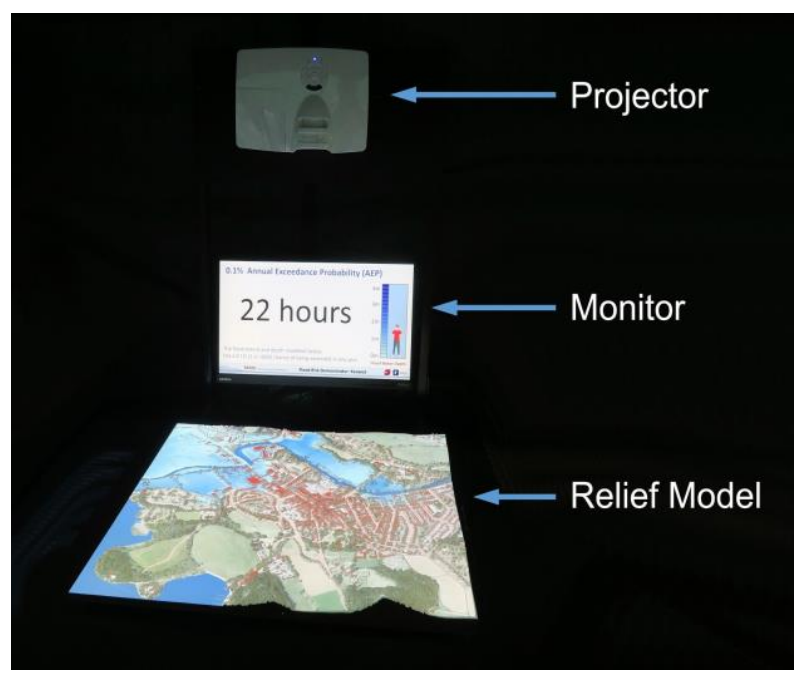

Figure 2. Typical Projection Augmented Relief Model (PARM) configuration

The texture maps used to augment relief models on Earth, such as maps or aerial photography (see Figure 2) help to emphasize the scale of the landscape involved, as they contain representations of familiar sized features such as fields, streets and even houses.

For Martian landscapes the texture maps related to land cover on Mars do not contain such features and the viewer has no direct experience of the landscapes to draw upon.

Projection-enhanced models present an opportunity to design projections that enhance the viewers' appreciation of spatial scale and promote a greater understanding of the nature of the Martian landscape.

This project required techniques that communicate the scale of a range of Martian landforms and where appropriate make connections to Earth-like geomorphology both in terms of scale and the processes that may have generated such landforms. PARM was considered a suitable technique for a number of reasons:

- It offered an engaging form of display suitable for groups of people rather than individual viewers

- It gave viewers a way of appreciating subtle differences in elevation and in slope due to direct human stereovision and the 'structure from motion' gained through moving around the models.

- It allowed development of dynamic content for protection over the model and display on-screen simultaneously, and creativity in the way projected images could convey scale in an unfamiliar landscape. Information on the screen could add descriptive context to general projections or display some kind of additional visualisation specific to a location concurrently highlighted on the model. 
A methodology was therefore developed which would use landscape models to represent a wide range of features at a number of scales for use at outreach events and in educational contexts.

\section{The Development of MarsCAPE}

In order to achieve the aims set out above, a prototype model was created which covered an area at a scale suitable for identification and comparison of fluvial landforms on Mars and Earth. This allowed for creating context through presenting recognisable processes and highlighting the differences that exist.

This 'Water' model was taken to a range of public engagement activities in order to gain feedback from different audiences with regards the learning objectives of MarsCAPE. This allowed initial examination of the suitability of such models to engage the public and also suggest features of Mars that would be interesting for other models, allowing exploration of different scales and landforms, as well as ideas for different projections on the models. The activities predominantly took place in the local area, organised by the University and the local branch of the British Science Association. They varied across types of audience, including events targeted at local school children, families and the general public, and student-focused workshop and careers events. As such, the range of events provided a platform for demonstrating MarsCAPE to large and diverse audiences of non-experts in different settings.

These outreach sessions had great value for helping to understand what interested people when presented with this kind of display, and how people would interact with them 'in the wild', rather than simply testing the rig in the lab. For example, at events targeting families and school children, often initial interactions came from the younger people, eager to 'play' with the model, but this often drew in the rest of their group; student groups and events targeted at adults often involved small groups of one or two people discussing the projections and pointing out interesting features. From these initial deployments in public settings, it was clear that audiences showed a willingness to learn from the projections and features highlighted on the model, which after creation of two further models led to the design of a more controlled deployment of all three to further examine how audiences react in terms of interest and understanding.

The success and informal feedback from these first events also led to the choice of focus of the two additional models: Fire and Wind. Model 2 focused on a larger area to visualise a volcano and its craters ('Fire'), and model 3 covered a smaller area to showcase sand dunes ('Wind'). In each of these themes, geomorphological features exist that occur both on Mars and Earth, again providing increased context. Figure 3 shows the relative size and location of each model on the surface of Mars, whilst the following sections describe each model: the geological phenomenon of interest and why, their size and scale, and how they were displayed.

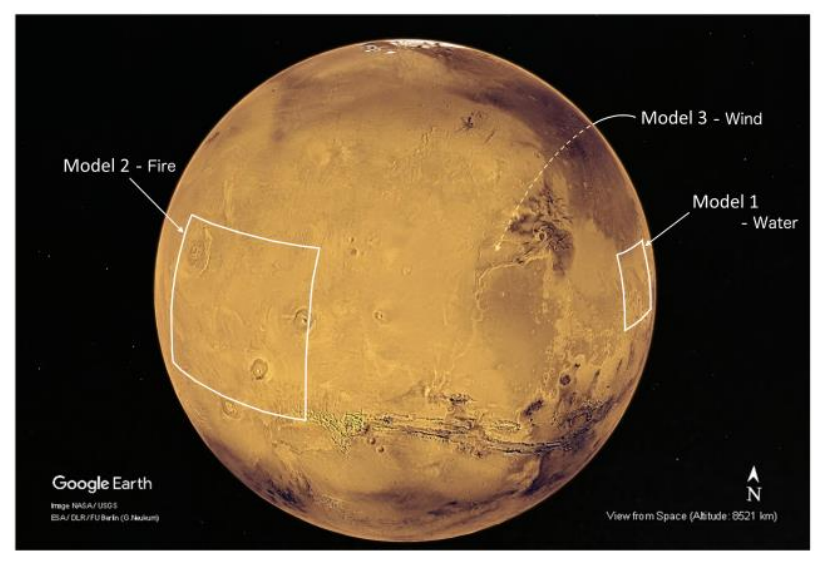

Figure 3. Relative positions of MarsCAPE models

\section{Model 1-Water}

The water model (Figure 4) centres around historical fluvial events that took place on the Martian surface. The central focus is the Ares Vallis outflow channel, thought to be formed by several flooding outflow events that occurred over a long period of geological time at decreasing surface levels [18]. The main feature of the model is a large, dried up channel or valley running in a northwest direction, progressively widening to open up into a delta like region. Its appearance is similar to major river flows on Earth (the Nile and its associated delta for instance). However, the width of the Ares Vallis delta dwarfs that of the Nile, and its peak outflow is many times that of the present-day Mississippi. In addition to the main channel, several large craters are visible on the model, along with 'islands' formed by fluvial events. Towards the northwest of the model is also the location of the Mars Pathfinder mission landing site (mars.nasa.gov/MPF), adding context to the mission aims and objectives.

The water model represents the middle scale of the three models, with its total area being comparable to that of Great Britain and Ireland. At this size, the Ares Vallis delta and several hundred kilometres of the channel can be represented. The model was created using a DTM dataset combining data from the Mars Global Surveyor (MGS) Mars Orbital Camera (MOC) and Mars Orbiter Laser Altimeter (MOLA), attaining a horizontal resolution of $\sim 10 \mathrm{~m}$ per pixel. In order to create a physical relief model the DTM data was cropped within ArcGIS and exported as a mesh, processed into machine paths for a Computer Numerically Controlled (CNC) milling machine to carve a model, measuring $60 \mathrm{~cm} \times 60 \mathrm{~cm}$, from model board. Milling was chosen over 3D printing because the model was quite large and did not have fine surface detail to represent. It was milled using a machine capable of movements less than $0.1 \mathrm{~mm}$.

The configuration of the PARM display featured the model placed flat on a table, a portable projector rig, and a monitor behind the model. To allow simple and rapid editing, content was delivered using two PowerPoint shows synchronised so that a transition on the model triggered a transition on the 
monitor. The content played on a continuous loop and featured a number of techniques designed to engage the audience with the nature and scale of the landscape:

- Animated sunrise effect projected onto the model brought out subtleties in relief that would otherwise be hidden with a fixed light source. This sequence of images was produced within a CAD package using the same terrain data used to produce the physical model. Images were rendered using progressively higher angle of illumination and the slowly morphed together as a projected sequence over the model.

- $\quad$ Relief shading combined with elevation colour ramp emphasised craters and delta-like features, with a graphic comparing the elevation range with that of Mount Everest on the monitor. Relief shading adds emphasis to more subtle physical features giving the impression they are illuminated from the side.

- Animated flood inundation and draining to highlight the subtle delta geomorphology and suggest water action as a process of landscape formation. This effect did not attempt to mimic water flow, instead using a simple rise and fall of water across the delta like feature to emphasise the channelized pattern. These images were rendered within a CAD package as with the sunrise effect.

- Geology map with legend on the monitor. In this case the monitor was used for more than simply labelling the projection, in this case it provided an explanation of the different rock types shown by coloured areas on the projected map.

- Map of the Nile delta projected over the model to suggest both scale and process. The map was positioning in such a way as to overlap the broad delta like feature visible on the model.

- Map of the UK projected over the model to offer a more familiar frame of reference. The map was positioning centrally to the model, in such a way that several large craters fell within England and Wales to provide an easy frame of reference for viewers to gauge the size of such features.

- Animated movement of a rover vehicle over the model with the passage of time displayed on the monitor in days and months from the date of the particular demonstration. At intervals along the route the 'rover' stopped, showed a cone of vision on the model, and a 3D perspective view of the terrain on the monitor.
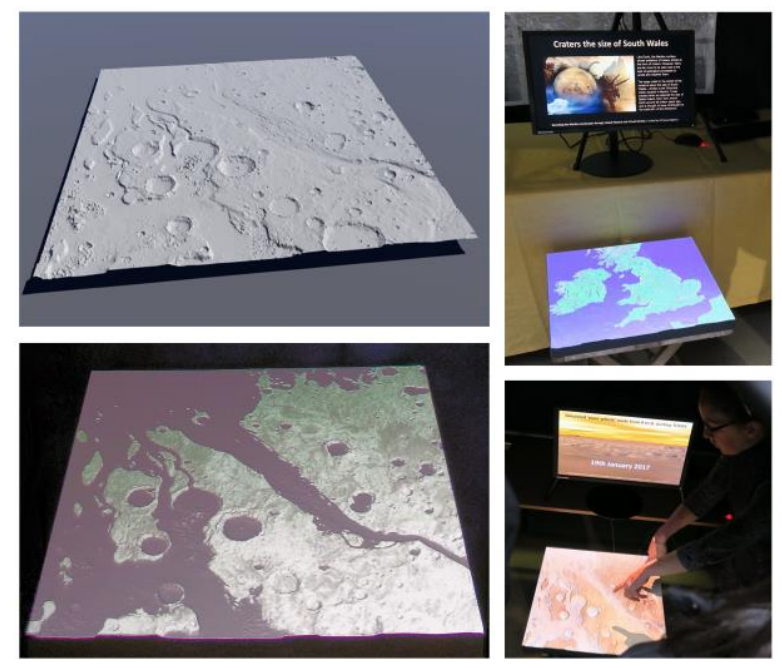

Figure 4. The water MarsCAPE model

\section{Model 2-Fire}

The fire model (Figure 5) centres around the historic volcanic activity that occurred on the surface of Mars. The area concentrates on the Tharsis Montes region, which contains the three volcanoes Ascraeus Mons, Pavonis Mons and Arsia Mons that lie in a straight line heading north east, and stretches out to the largest known volcano in the solar system, Olympus Mons. They are examples of shield volcanoes that are also found on Earth, named for their low profile caused by low viscosity lava travelling farther across the surface. Despite the similarities in their formation on both planets however, differences in scale exist due the contrasting atmospheres and gravity of each planet [19]. In addition to the volcanoes featured, a section of the Valles Marineris canyon system is also visible on the model, which again can be compared to similar canyon systems on Earth.

In order to display such large features (Arsia Mons alone has a diameter of $460 \mathrm{~km}$ ) the model needs to cover a large section of the planetary surface. To achieve this the model was created using a blended DTM/DEM dataset derived using data from both the Mars Express High Resolution Stereo Camera (HRSC), and MOLA. Instead of being milled, 3D printing was used to ensure the finer details of canyons and small craters were visible, and a planetary curve was added to emphasise the large scale of the area represented $(\sim 1 / 12$ th of the entire planet surface). It was printed in plaster powder at an approximate resolution of $600 \mathrm{dpi}$ with a layer thickness of $0.1 \mathrm{~mm}$.

The content displayed over the model and monitor featured similar themes to model 1 but focussing on Olympus Mons:

- $\quad$ Sunrise effect revealing imagery from the Viking mission in the 1970s. Given that the curvature of the planet was represented in this model, the changing illumination angle helped to pick out the fine detail of craters and canyons at various locations around the surface of the model. 
- A map of Europe projected over the model, with France placed over Olympus Mons. The outer edge of Olympus Mons was aligned with the coastline and borders of France, resulting in Italy being towards the lower right quarter of the model, providing a clear frame of reference for judging the scale of the whole model as well as Olympus Mons.

- A map of Western USA drawing attention to the large size of Valles Marineris compared to the Grand Canyon. In this case because Valles Marineris was so much larger than the Grand Canyon, and therefore the majority of it was 'off the model', the monitor was used to show an image of what was projected on the model but also the wider area, showing the viewer the size of this feature relative to the area represented on the physical model.

- Cross-sections on the monitor displayed direct comparisons of the relative size of Martian landscape features compared to familiar features on Earth, marking the locations of the sections using lines projected onto the model. These sections included the Grand Canyon shown within the Valles Marineris and Olympus Mons towering over Mount Everest.
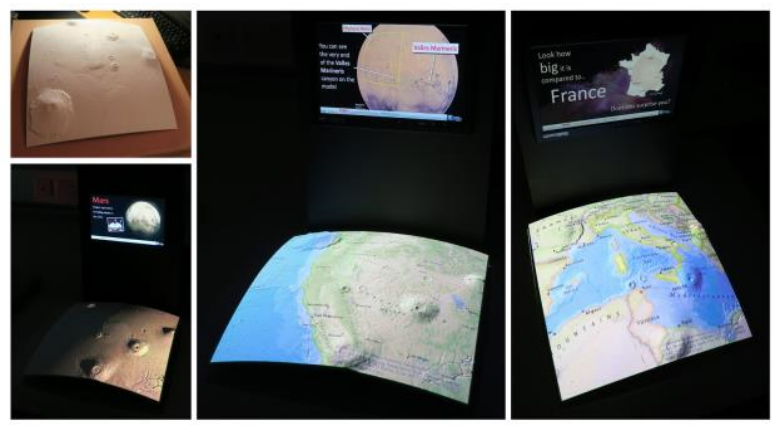

Figure 5. The fire MarsCAPE model

\section{Model 3 - Wind}

Finally, the wind model (Figure 6) represents aeolian processes on the Martian surface, and unlike the other two models demonstrates activity that to an extent is still occurring. It focuses on sand dunes, specifically Barchan-type, that appear ubiquitously across the planetary surface. Barchan dunes are found widely on both Earth and Mars, and the morphology of their limbs (or arms) can give clues regarding the direction of the winds that formed them, their collisions, and the incline of the surface on which they migrate. Whilst dune migration occurs on both planets, it tends to happen at a much slower rate (less than a metre a year) on Mars due to the thinness of the atmosphere [20]. In addition to the dunes, dominating the centre of the model is an impact crater. Such impact events are still occurring on the Martian surface, and the ejecta of the impact can be clearly seen on the model as a smooth region where the sand dunes have been effectively flattened.

The features highlighted on the wind model are at a much smaller scale than those of the other two models, with sizes in the region of hundreds of metres rather than kilometres. As such, the area covered by the model is much smaller $(\sim 4.2 \mathrm{~km}$ total diameter), and the data used to create it needed to be of a much greater resolution. The model was therefore constructed using a DTM dataset created with data from Mars'

Reconnaissance Orbiter's (MRO) High Resolution Imaging Science Experiment (HiRISE) instrument. HiRISE images and associated DTMs are some of the highest quality remotely sensed datasets ever taken of Mars, achieving a resolution of $\sim 1 \mathrm{~m}$ per pixel, and so reveal high levels of detail. As with the water model, the wind model was produced through the milling of model board. The model was used without projection but was accompanied by questions promoting inquiry related to wind process and form. This also allows a comparison with projection-enhanced models to explore whether people interacted with it in different ways or made suggestions about how it could be usefully augmented.
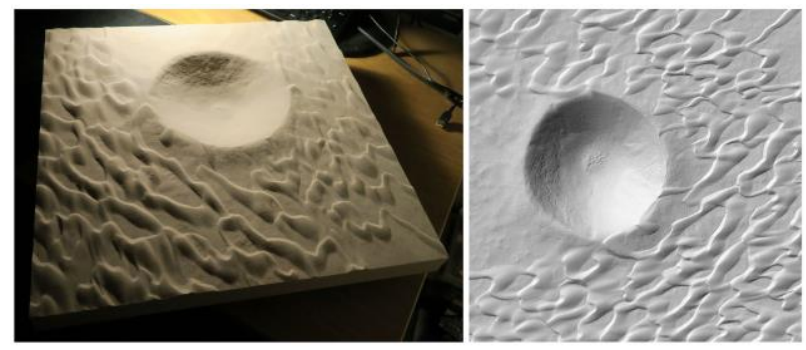

Figure 6. The wind MarsCAPE model

\section{Model Deployment in a Public Setting}

All three models were presented at a workshop at the UK National Space Centre as part of a week-long space training programme provided by the National Space Academy for engineering undergraduates from Beijing. Participants were 11 males and 10 females, with an average age of 20. The MarsCAPE team facilitated an activity using the three models to identify and discuss potential locations for landing and habitation. The activity was designed to evaluate how each model and the overall MarsCAPE setup could help achieve the students' learning objectives. The workshop setup is now described in more detail.

\section{Strategy for Evaluation}

Participants were first given a questionnaire which asked them to rate their level of knowledge and interest (on a 7 point Likert-like scale) in various topics related to information presented by the models: planetary science, geomorphology, the Martian Landscape, space exploration, 3D modelling, Satellite technology, and how data from satellite imaging is used. They were then split into three groups of roughly equal 
size and were given approximately 20 minutes to interact with each model in turn, considering what each model could tell them about the surface of Mars and how scientists might use that information in their considerations for landing sites and potential habitation. The structure of the interactions were left fairly open so that participants could work with the models however they chose, but each group was given 2 worksheets to complete for each model, and the event facilitators also spent time with each group, asking questions where relevant to prompt discussion.

One worksheet was a SWOT-style (Strengths, Weaknesses, Opportunities, and Threats) grid. This was a quick and interactive way to get participants to consider the positives and negatives of both the models themselves and the sites they represent. Participants were asked to consider the strengths and weaknesses of each models for studying Mars, and then the opportunities and threats of potential landing or habitation in the areas the models were illustrating. The other worksheet allowed groups to draw their own diagrams of each model and mark what they felt were important features of each terrain. They were given the scale of the model, as well as space for a legend and for sketching. The order of completion of the worksheets was left up to the group.

After each group had spent time with all three models, participants were brought back together for a brief discussion period followed by a second questionnaire. This repeated the ratings questions from the first questionnaire to monitor any change, but also used free-text responses to find out which model was the favourite, and why, what they considered the main issues facing scientists planning for landing and habitation of Mars, and what further information they would like on the models. They were also presented with a series of statements on a 7-point Likert-like scale related to the learning objectives of the models and the session. Finally they were asked to mark on a bipolar scale (e.g. Enjoyable/Annoying, Difficult/Easy) how they felt about the session overall.

\section{Participant Responses to Interacting with the Models}

The participants overwhelmingly chose the water model as favourite $(81.0 \%)$. The reasons given for this preference often surrounded the scale of the model, particularly for the specific session tasks, and for allowing a good balance between detailed information and an overview of the area. The presentation of varied information was also mentioned by several, including in particular seeing the journey a rover might take, and comparison to the size of places on Earth to illustrate scale. Historical changes to the surface and information about altitude, sunlight, and other factors that allowed them to consider the potentials for landing and habitation (e.g. chances of finding water) were also popular. Several people also felt that it was the most attractive model, and commented on the colourful projections. Identified strengths included a good range of useful information, including comparison data for visualisation of scales and distances. Weaknesses revolved around the level of detail available on the model. In terms of human habitation, this model seemed good for initial planning stages of a mission, but less good for a detailed study of a chosen area.

Figure 7 provides an example of the areas of the model that were highlighted. The groups tended to mark more features for model 1 than the others, including the slope of the land, where resources might be found, and areas that might be more protected from the elements. All groups provided at least one suggestion for a landing or habitation site. One group provided several suggestions for habitation, also considering where water exploration might be fruitful, and marking the rover route shown on the projection to help with this decision.

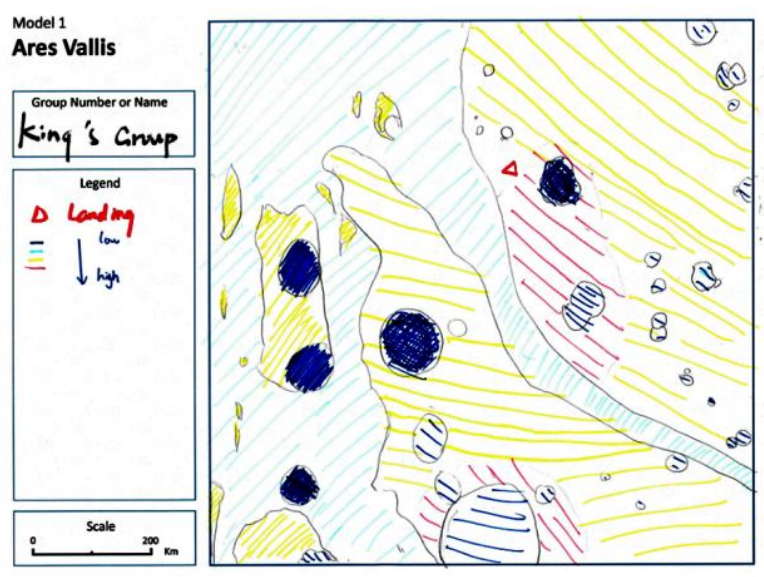

Figure 7. An example diagram for Water Model

The fire model was the favourite for just $9.5 \%$ of the participants, who felt that it was the most interesting for considering the issues raised in the session. SWOT responses indicated that the strengths of the model and opportunities for human habitation both predominantly focused on the range of landmarks that were visible on the model, and the ability to judge scale and distance due to the curvature, despite it being missing some potentially important data and detail.

Figure 8 provides an example of the areas of the model that were highlighted. Two of the groups indicated potential landing sites, with one group pointing out that theirs was "among three mountains, where the land is flat, which is conducive to landing and survival". They also indicated areas they felt would be bad for landing, in and around craters, and an area that would be good for habitation in a relatively flat part of the model. Another group also picked this area for both landing and habitation. All groups marked the main craters and landforms such as canyons to help them position their decisions on the map. 


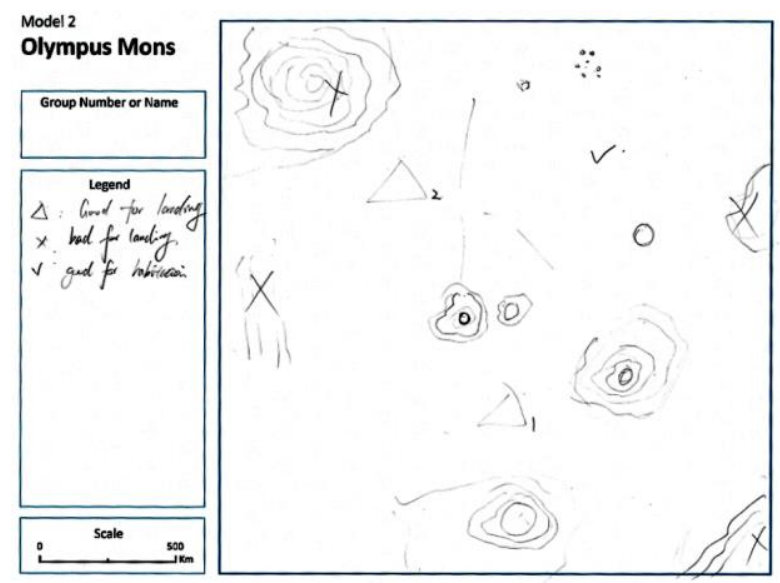

Figure 8. An example diagram for Fire Model

The wind model was the favourite for just $9.5 \%$ of the participants, who liked the amount of information provided, and felt it was easiest to distinguish landmarks. The main strengths of this model identified through the SWOT worksheets were the amount of detail due to the small scale, and the feeling of tactility and greater accuracy. However, due to the lack of projections the main weaknesses surrounded the lack of information provided, including historical and climate data. Opportunities for landing sites included appreciation for being able to plan the size of a base and the scale of rover routes, as well as the potential for locating good resources. However potential threats included wind and sandstorms, and they also noted a lack of choice in where to land or explore due to the small scale.

Figure 9 provides an example of the areas of the model that were highlighted. All 3 groups suggested landing sites on their maps, as well as estimating wind direction. The smaller scale appears to have allowed for more detailed planning of a habitation zone. One group also suggested sites for mining, a science lab, and even a shopping mall! The students also suggested some projections that would be useful on this model, including animated wind arrows.

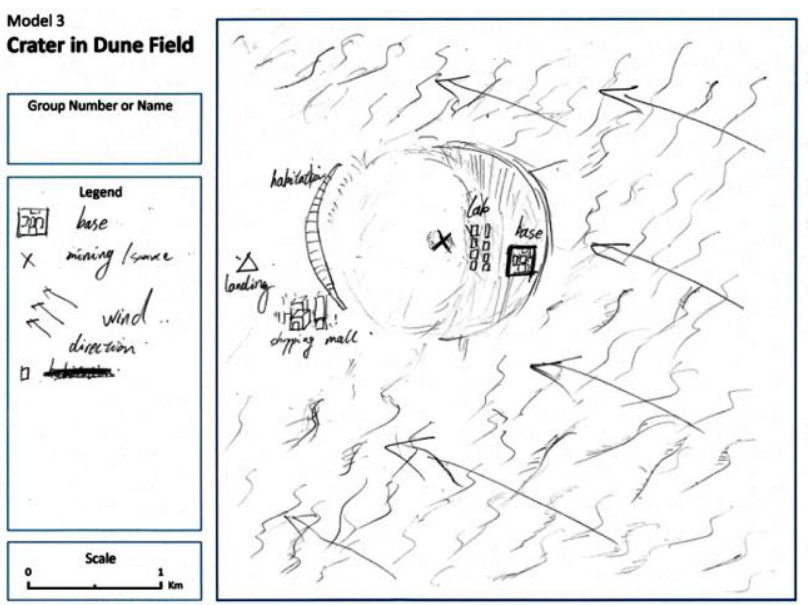

Figure 9. An example diagram for Wind Model

During the post-session questionnaire, participants were asked what further information on the three models they would like to see. There were 32 suggestions in total, of which the most common were temperature data (5) and height information (4). Some wanted other types of historical data such as atmospheric information, weather, sandstorms, wind direction, and surface changes year to year. Some wanted more current geological or topographical information, as well as information about the soil structure or possible water or natural resources. Finally, general information like a clearer indication of scale, more colourful projections, more explanations of features and place names were also requested. Two people made suggestions for improvement in the actual technology in the rig: having the projector respond to moving the model, and a touchscreen to make information interactive.

\section{Perceived Knowledge and Interest}

The results from questionnaires showed that participants' perceived knowledge of the various factors involved in the session consistently increased (Table 1). Initial knowledge was mostly quite low, with the highest levels for space exploration (3.6) and lowest for geomorphology (2.4). Wilcoxon signed ranks tests show that increase in knowledge was significant for almost all of the factors, except space exploration and satellite technology. As seen in Table 3, participants felt that they learnt a lot about the surface of Mars and the issues involved in habitation and landing. The models helped them to understand and were a useful tool to teach people about and to explore the surface of Mars.

Interest in each factor was predominantly high to begin with; highest initial interest was also in space exploration (5.8) and lowest in geomorphology (3.6). Average interest mostly increased slightly, but only 3D modelling was significant (Table 2). However, they did report that their interest in the topics covered had increased (Table 3). 
Table 1. The difference in average scores for level of knowledge before and after the session (* indicates the difference is significant)

\begin{tabular}{|llllll|}
\hline Factor & $\begin{array}{l}\text { Average knowledge } \\
\text { (before session) }\end{array}$ & $\begin{array}{l}\text { Average knowledge } \\
\text { (after session) }\end{array}$ & Difference & $\begin{array}{l}\text { Wilcoxon } \\
\text { signed ranks (Z) }\end{array}$ \\
\hline Planetary science & 3.3 & 3.7 & +0.4 & -2.138 & $.033^{*}$ \\
\hline Geomorphology & 2.4 & 3.5 & +1.1 & -3.372 & $.001^{*}$ \\
\hline Martian landscape & 2.6 & 3.9 & +1.3 & -3.425 & $.001^{*}$ \\
\hline Space exploration & 3.6 & 4.1 & +0.5 & -1.838 & .066 \\
\hline 3D modelling & 3.0 & 3.7 & +0.7 & -2.157 & $.031^{*}$ \\
\hline Satellite technology & 3.0 & 3.5 & +0.5 & -1.805 & .071 \\
\hline Satellite data use & 2.8 & 3.7 & +0.9 & -2.301 & $.021^{*}$ \\
\hline
\end{tabular}

Table 2. The difference in average scores for level of interest before and after the session (* indicates the difference is significant)

\begin{tabular}{|llllll|}
\hline Factor & $\begin{array}{l}\text { Average interest (before } \\
\text { session) }\end{array}$ & $\begin{array}{l}\text { Average interest (after } \\
\text { session) }\end{array}$ & Difference & $\begin{array}{l}\text { Wilcoxon } \\
\text { signed ranks (Z) }\end{array}$ & P-value \\
\hline Planetary science & 5.4 & 5.3 & -0.1 & -0.275 & .783 \\
\hline Geomorphology & 3.6 & 4.2 & +0.6 & -1.669 & .095 \\
\hline Martian landscape & 4.8 & 4.9 & +0.1 & -0.504 & .614 \\
\hline Space exploration & 5.8 & 5.6 & -0.2 & -1.667 & .096 \\
\hline 3D modelling & 4.6 & 5.1 & +0.5 & -2.027 & $.043^{*}$ \\
\hline Satellite technology & 4.9 & 5.0 & +0.1 & -0.443 & .658 \\
\hline Satellite data use & 5.1 & 5.2 & +0.1 & -0.540 & .589 \\
\hline
\end{tabular}

Table 3. Model \& session statements average level of agreement (1=I completely disagree, $7=\mathrm{I}$ completely agree)

\begin{tabular}{|lc|}
\hline Statement & Average \\
\hline I learnt a lot today about the surface of Mars & 5.8 \\
\hline $\begin{array}{l}\text { I understand more now about the issues } \\
\text { involved in the habitation of Mars }\end{array}$ & 4.7 \\
\hline $\begin{array}{l}\text { The models helped me to understand more } \\
\text { about the surface of Mars }\end{array}$ & 6.3 \\
\hline $\begin{array}{l}\text { I understand more now about the problems } \\
\text { involved in landing on Mars }\end{array}$ & 5.1 \\
\hline $\begin{array}{l}\text { The models are a useful tool to teach people } \\
\text { about the surface of Mars }\end{array}$ & 6.6 \\
\hline $\begin{array}{l}\text { The models were a good way to explore the } \\
\text { surface of Mars }\end{array}$ & 6.0 \\
\hline $\begin{array}{l}\text { After today's session, I am more interested in } \\
\text { the topics covered }\end{array}$ & 6.3 \\
\hline
\end{tabular}

The participants were given the opportunity to add any other comments about their learning and interest during the session. Of the 20 comments, ten related to learning, three to interest, and six to the session as a whole. With regards the session overall, one participant felt it was quite hard and 4 suggested that more time would have been useful. Another suggested that it would be useful to have further teaching around theory after having used the models. Participants felt they had learnt a lot about Mars in general including climate and geology, and were more interested in future missions: "Today's events have greatly increased my understanding of Mars and made me look forward to China's Mars exploration project". Some also mentioned learning about the use and combination of data types, and 3D modelling. The participants were also asked to list the main issues for scientists when planning for landing and habitation of Mars. The 59 issues could be grouped into: global conditions, for example atmospheric pressure and climate (19); local conditions, such as storms and other extreme weather conditions (11); surface features at landing sites (10); concerns about resources such as food and water (8); life support and energy supplies (6); and considerations of wider scientific exploration, such as expense and where to explore (5).

\section{Discussion}

The main aims of the MarsCAPE project are to engage the public with space and human space exploration; to improve understanding of the Martian surface, missions to Mars, and the practicalities of space exploration; and to provide public access to planetary data that they otherwise would not necessarily engage with. This section will reflect on these aims through formal feedback from the event participants, and more informal observations by the MarsCAPE investigators. 


\section{Using MarsCAPE for Education and Engagement: Participant Feedback}

The participants of the final workshop overwhelmingly chose the water model based on data from MOC and MOLA, as the favourite. This model focuses on the Ares Vallis outflow channel, a large dried up valley running northwest to a delta. This model was the middle of the three scales used for the models, approximately $10 \mathrm{~m}$ per pixel, covering a size comparable to Great Britain and Ireland. Participants liked this model best especially due to the scale and the varied information that was presented, as well as the attractiveness of the projections. Participants were able to use all three models to effectively discuss what each could tell them about the surface of Mars and how scientists might use that information in their considerations for landing sites and potential habitation. Model 1 was felt to be useful due to the comparison data that was presented, allowing easier visualisation of scales and distances, but participants also would have liked more detail. This model proved useful for consideration of the initial planning stages of a mission, but was less good for a detailed study of a chosen area. Participants thought that the fire model based on MGS, HRSC, and MOLA data surrounding Olympus Mons, had a good range of landmarks visible, allowing them to judge scale and distance due to the curvature, despite it being missing some potentially important data and detail. They tended to focus on the benefits of the different land formations but were concerned about the lack of data from other sources such as temperature. Participants found the main strengths of the wind model based on data from the HiRISE camera to be related to the amount of detail afforded by the small scale. They also like the tactile nature of the model due to a lack of projections, but felt that this meant they could get much less information from it.

Participants self-rated knowledge of the subjects that the models were designed to educate about were originally fairly low, with a significant increase by the end of the session in almost all areas: planetary science, geomorphology, Martian landscape, 3D modelling, and the use of satellite data. Selfrated knowledge did not increase for space exploration and satellite technology, perhaps because these subjects are seen to be less focussed or relevant to the examination of a specific planet's surface. Participants did feel that they learnt a lot about the surface of Mars and the issues involved in habitation and landing on Mars. They also felt the models were useful for understanding, teaching, and exploring the surface of Mars. Although participants indicated that their interest had increased through the session, self-ratings of interest were high from the start, and only interest in 3D modelling was significantly increased by the use of the models. Participants also indicated that they would like to have had more time with the models. These results suggest the models are a good tool for both education and engagement; people enjoy interacting with them and they come away feeling that they have learnt something. This includes a natural learning process from interacting with the models as well as the instructional learning from projections and screen-based text. People focus on and pick up on particular things based on their own specific interests.

When asked about the main issues for scientists when planning for landing and habitation of Mars, participants came up with a wide range of suggestions, from hyper local considerations to awareness of wider issues of scientific exploration. To help answer the question, two participants made suggestions for improvement in the actual technology in the rig: having the projector respond to moving the model, and a touchscreen to make information interactive. Additional information that would be useful for all three models primarily fell into three main areas: extra geological data, historical (mostly weather) data, and general information such as scales and place names. One team specifically suggested adding animated wind arrows to the models.

\section{Investigator Observations}

Beyond the more formal feedback gathered from the participants of the event, the MarsCAPE investigators also made informal observations regarding the effectiveness of the system for engagement and learning.

Overall it was clear the three models are an engaging form of display, suitable for groups of observers and not just individuals. As such, it allowed for a more collaborative approach to be taken, where participants could discuss the information being displayed and its use. MarsCAPE gave viewers a way of appreciating subtle differences in the elevation and slope of Martian landforms both through direct human stereovision and 'structure from motion' gained through moving around the models. MarsCAPE gave the research team a creative environment for exploring dynamic content for projection over the model and display on a screen simultaneously. This content effectively conveyed scale by relating the Martian landscape to a familiar frame of reference, increasing understanding and engagement. Information on the screen added descriptive context to projection effects or could display visualisations specific to a highlighted place on the model such as the panoramas of the hypothetical rover journey, adding interest and further context.

\section{Conclusion}

The broad aim of MarsCAPE was to engage the public with the planet Mars, and in doing so improve understanding and increase accessibility to planetary data beyond what is possible with traditional on-screen visualisations. Through running a workshop at the UK National Space Centre with 21 undergraduate students without specific planetary expertise, it is clear that MarsCAPE was at least somewhat successful in doing this.

The participants' self-rated knowledge significantly increased in a number of planetary areas including the Martian landscape, geomorphology and satellite data, and they felt the MarsCAPE models were useful for increasing understanding, teaching and exploration. The models also facilitated a natural 
learning process, where participants picked-up and focussed on ideas without instruction. Beyond the formal feedback, participant comments demonstrated a nuanced understanding regarding the importance of scale, recognising that the different models conveyed different granularities of data allowing for different levels of inferred information. Investigator observations noted the strength of MarsCAPE in providing a more group-orientated, collaborative approach to learning about the Martian surface, allowing viewers to appraise slope and elevation by moving around the models.

Models that use the PARM approach, like MarsCAPE, have the potential to provide an engaging, educational way of accessing planetary data that go beyond the capabilities of onscreen visualisations. Different scales and processes can be displayed, giving context to the features that exist and how they compare to similar examples on Earth. However, there is a need for future investigations to measure the public engagement value of such systems in a more formal way, including the role they could play as a permanent part of an exhibition space.

\section{References}

[1] Y. Guo and R. W. Farquhar, "New Horizons Mission Design," Space Sci. Rev., vol. 140, no. 1, pp. 49-74, Oct. 2008, doi: 10.1007/s11214-007-9242 $\mathrm{y}$.

[2] H. V. Hendrix, G. Slusser, and E. S. Rabkin, Visions of Mars: Essays on the Red Planet in Fiction and Science. McFarland, 2014.

[3] J. Parnell et al., "Searching for Life on Mars: Selection of Molecular Targets for ESA's Aurora ExoMars Mission," Astrobiology, vol. 7, no. 4, pp. 578-604, Aug. 2007, doi: 10.1089/ast.2006.0110.

[4] M. E. Schwamb et al., "Mapping Mars' Southern Springtime Winds and Seasonal Polar Fans with Planet Four," presented at the AAS/Division for Planetary Sciences Meeting Abstracts \#50, 2018, vol. 50, p. 300.01.

[5] J. Raddick et al., "Galaxy Zoo: Motivations of Citizen Scientists," Astron. Educ. Rev., vol. 12, no. 1, pp. 1-41, 2013.

[6] K. Masters et al., "Science Learning via Participation in Online Citizen Science," ArXiv160105973 Astro-Ph Physicsphysics, no. A07, pp. 1-33, Jan. 2016.

[7] A. $\mathrm{Na}$ et al., "Planetary Cartography and Mapping: where we are Today, and where we are Heading For?," ISPRS - Int. Arch. Photogramm. Remote Sens. Spat. Inf. Sci., vol. 62W1, pp. 105-112, Jul. 2017, doi: 10.5194/isprsarchives-XLII-3-W1-105-2017.

[8] R. B. Schultz, J. J. Kerski, and T. C. Patterson, "The Use of Virtual Globes as a Spatial Teaching Tool with Suggestions for Metadata Standards," $J$. Geogr., vol. 107, no. 1, pp. 27-34, 2008.

[9] G. Priestnall, J. Gardiner, J. Durrant, and J. Goulding, "Projection Augmented Relief Models (PARM): Tangible Displays for Geographic Information," in Proceedings from Electric Visualisation and the Arts, London, UK, 2012, pp. 180-187.

[10] J. Sprinks, J. Wardlaw, and G. Priestnall, "MARSCAPE: Exploring the Martian Landscape through PARM (Projected Augmented Relief Model)," presented at the EGU General Assembly Conference Abstracts, 2017, vol. 19, p. 16620.

[11] K. Bladin et al., "Globe Browsing: Contextualized Spatio-Temporal Planetary Surface Visualization," IEEE Trans. Vis. Comput. Graph., vol. 24, no. 1, pp. 802-811, Jan. 2018, doi: 10.1109/TVCG.2017.2743958

[12] I. Bishop, E. Lange, and I. D. Bishop, Visualization in Landscape and Environmental Planning: Technology and Applications. Taylor \& Francis, 2005 .

[13] S. R. J. Sheppard, Visual simulation: a user's guide for architects, engineers, and planners. New York: Van Nostrand Reinhold, 1989.

[14] S. R. J. Sheppard, "Guidance for crystal ball gazers: developing a code of ethics for landscape visualization," Landsc. Urban Plan., vol. 54, no. 1, pp. 183-199, May 2001, doi: 10.1016/S0169-2046(01)00135-9.

[15] G. A. Rothrock, "The Musee des Plans-Reliefs," Fr. Hist. Stud., vol. 6, no. 2, pp. 253-256, 1969, doi: 10.2307/286168.

[16] A. W. Pearson, "Allied Military Model Making during World War II," Cartogr. Geogr. Inf. Sci., vol. 29, no. 3, pp. 227-242, Jan. 2002, doi: $10.1559 / 152304002782008468$.

[17] S. de Chadarevian and N. Hopwood, Models: The Third Dimension of Science. Stanford, USA: Stanford University Press, 2004.
[18] N. Warner, S. Gupta, J.-P. Muller, J.-R. Kim, and S.-Y. Lin, "A refined chronology of catastrophic outflow events in Ares Vallis, Mars," Earth Planet. Sci. Lett., vol. 288, no. 1, pp. 58-69, Oct. 2009, doi: 10.1016/j.epsl.2009.09.008.

[19] L. Wilson and J. W. Head, "Evidence for a massive phreatomagmatic eruption in the initial stages of formation of the Mangala Valles outflow channel, Mars," Geophys. Res. Lett., vol. 31, no. 15, 2004, doi: 10.1029/2004GL020322.

[20] S. Silvestro et al., "Pervasive aeolian activity along rover Curiosity's traverse in Gale Crater, Mars," Geology, vol. 41, no. 4, pp. 483-486, Apr. 2013, doi: 10.1130/G34162.1

James Sprinks is a Research Fellow at Nottingham Trent University's Medical Design Research Group. His research considers how citizen science platforms can be designed and implemented to both ensure that the data generated is in a usable format and scientifically robust, while still maintaining a user experience that results in volunteers enjoying and learning from the process.

Liz Dowthwaite is a Research Fellow in the Horizon Digital Economy Research Institute at the University of Nottingham. She has a multi-disciplinary research background in Psychology and Human Factors, and her research interests include motivations in online crowd systems (crowdfunding, social media, citizen science); the effects of the online world on psychological wellbeing; and engagement and learning in science communication.

Gary Priestnall is an Associate Professor at the University of Nottingham. His research interests are in the area of Geographic representation and visualisation. This includes the design of various forms of geographic situated display, landscape visualisation, and how geographic information is displayed and captured via mobile devices.

Jessica Wardlaw carried out this work as a Postdoctoral Research Fellow in the Faculty of Engineering at the University of Nottingham, UK. Her research applies her expertise in Geographical Information Systems and HumanCentred Design to her interest in Citizen Science, and the participation of non-professionals in professional scientific activities. 
